\title{
Management of pediatric head and neck rhabdomyosarcoma: A case-series of 36 patients
}

\author{
JOANNA RADZIKOWSKA ${ }^{1}$, WOJCIECH KUKWA ${ }^{1}$, ANDRZEJ KUKWA ${ }^{2}$, ANNA M. CZARNECKA ${ }^{3}$, \\ MACIEJ KAWECKI ${ }^{3}$, FEI LIAN ${ }^{4}$, CEZARY SZCZYLIK $^{3}$ and ANTONI KRZESKI ${ }^{1}$ \\ ${ }^{1}$ Department of Otorhinolaryngology, Faculty of Medicine and Dentistry, Medical University of Warsaw, Warsaw 00-739; \\ ${ }^{2}$ Department of Otolaryngology and Head and Neck Disease, University of Varmia and Mazury, Olsztyn 10-082; \\ ${ }^{3}$ Department of Oncology and Molecular Oncology Laboratory, Military Institute of Medicine,Warsaw 04-141, Poland; \\ ${ }^{4}$ Department of Urology, Emory University School of Medicine, Atlanta, GA 30322, USA
}

Received August 22, 2015; Accepted May 19, 2016

DOI: $10.3892 / \mathrm{ol} .2016 .5072$

\begin{abstract}
Rhabdomyosarcoma (RMS) is the most common soft tissue sarcoma in the pediatric population. In $35 \%$ of cases, RMS develops in the head and neck $(H \& N)$ region, and only combined therapy is recognized as a curative treatment. However, recent advances in skull base and reconstructive surgery, along with microsurgery and endoscopic surgery, have strengthened the role of surgery as an important part of RMS treatment. In the present study, 36 pediatric RMS cases (24 males and 12 females) were analyzed after surgical treatment. The average age at diagnosis was 7 years. In total, $67 \%$ of tumors were localized in the parameningeal region. Alveolar RMS was the most common histopathological type. A total of 16 patients were treated due to disease recurrence or a previous non-radical surgical procedure, while 19 cases had inductive chemotherapy and/or radiotherapy preceding surgical treatment due to locally advanced disease. In 1 case, only diagnostic biopsy was performed. It is recommended that the management of H\&N RMS is interdisciplinary from the beginning. Extensive surgical dissection in the $\mathrm{H} \& \mathrm{~N}$ region for RMS may result in severe cosmetic defects and functional impairment; thus, these risks should be considered during treatment planning, and the surgical approach should be based on the individual characteristics of each patient.
\end{abstract}

\section{Introduction}

Rhabdomyosarcoma (RMS) is the most common soft tissue sarcoma in children, and represents $4.5 \%$ of

Correspondence to: Dr Joanna Radzikowska, Department of Otorhinolaryngology, Faculty of Medicine and Dentistry, Medical University of Warsaw, 19/25 Stepinska Street, Warsaw 00-739, Poland

E-mail: jwronska@poczta.onet.pl

Key words: surgery, rhabdomyosarcoma, head and neck cancer, sarcoma, neoplasm all pediatric malignancies (1-3), with an incidence of 4.5/1,000,000 cases (4). Although the majority of RMS cases arise sporadically, RMS may develop as a result of Li-Fraumeni syndrome (tumor protein p53 mutations), Beckwith-Wiedemann syndrome (11p15 defects), von Recklinghausen disease (neurofibromatosis type 1 mutations), cardiofaciocutaneous syndrome (B-Raf mutations) and Noonan syndrome [rat sarcoma (RAS)/mitogen-activated protein kinase signaling pathway mutations) (4). It has been reported that $>1 / 2$ of patients are $<10$ years old when diagnosed $(5,6)$. RMS has a bimodal age distribution, with a first peak of incidence between the ages of 2 and 6 years, and a second peak of incidence between the ages of 10 and 18 years $(1,7)$. RMS occurs slightly more often in males than in females, but there is no such difference in RMS that originates from the head and neck (H\&N) region (5). Nearly $35 \%$ of all RMS tumors develop in the H\&N region $(5,6)$. Within the H\&N type, it is clinically useful to divide RMS into three distinct categories: i) Orbital, ii) superficial and iii) parameningeal (8). Of all H\&N RMS cases, $44.0 \%$ are localized in the parameningeal region, which includes the nasal cavity, nasopharynx, paranasal sinuses, middle ear, infratemporal and pterygopalatine fossa (5), while $25.6 \%$ of H\&N RMS cases occur in the orbital region (5).

RMS develops from embryonic mesenchyme with the potential to differentiate into skeletal muscles (9). RMS may be divided into three major subtypes: i) Embryonal, ii) alveolar and iii) pleomorphic (10). Embryonal RMS develops as a result of oncogenic mutations involving the anaplastic lymphoma kinase, RAS, fibroblast growth factor receptor 4, phosphatidylinositol-4,5-bisphosphate 3-kinase catalytic subunit alpha or catenin-cadherin-associated protein beta 1 genes (4). By contrast, alveolar RMS arises as a consequence of the translocation between the forkhead box protein $\mathrm{O} 1$ transcription factor gene (which is located on chromosome 13) and either the paired box (PAX)3 transcription factor gene on chromosome 2 or the PAX7 gene on chromosome 1, and mutations in the v-Myc avian myelocytomatosis viral oncogene neuroblastoma derived homolog and the c-Met genes (4). Both RMS prognosis and treatment depend on the histopathological subtype (11). Of all patients with H\&N RMS, >1/2 
have embryonal RMS, which is a favorable prognostic factor, whereas the alveolar subtype carries a poorer prognosis (12). Other RMS negative prognostic factors include parameningeal localization, presence of distant metastases, non-radical primary surgical procedure, tumor size $>5 \mathrm{~cm}$, age at diagnosis $>10$ years old and time to tumor relapse (1).

Oncological advancements during the last decades have improved RMS treatment outcomes substantially (13). During the early 20th century, surgery was the only available therapy for RMS $(13,14)$. Radical excision was the standard of care, and survival rates were poor, with survival being $7-70 \%$ at that time (9).

Survival of children with RMS has improved significantly during the past 30 years (11). This improvement may be attributed to the development of multimodal therapy combining surgery, radio- and chemotherapy (11). According to the Intergroup Rhabdomyosarcoma Study (IRS) IV, the 5-year RMS survival increased from $25 \%$ in 1970 to $73 \%$ in $2001(15,16)$, but at the same time, almost $15 \%$ of children with RMS present with metastatic disease (group IV), and their prognosis has not improved significantly over the last 20 years (9). Radiation therapy is generally reserved for those patients with high-grade, unresectable tumors, and as adjuvant therapy following resection with cancer-positive surgical margins (4). Recent advances in radiation therapy include intensity modulated radiation therapy, specific computer-modulated radiotherapy and up-front radiotherapy prior to chemotherapy treatment (9).

In conjuction, chemotherapy is the backbone of therapy for RMS patients (4). The three-drug combination of vincristine, dactinomycin and cyclophosphamide (termed VAC) is considered the standard treatment for these patients. The D9803 and CWS-91 trials, and the MMT-98 and IRS IV studies, have all demonstrated that the addition of other agents, including ifosfamide, etoposide or topotecan, does not improve clinical outcomes in terms of overall response rate, overall survival (OS) and progression-free survival, compared with standard VAC $(4,9)$. Recently, combined therapy based on multidrug chemotherapy, advanced radiotherapy and more advanced surgical procedures (including skull base procedures, microsurgery and endoscopic and reconstructive surgery) have all contributed to an increase in survival (17). The formation of study groups such as the IRS (now known as the Children's Oncology Group), the International Society of Pediatric Oncology, the German Cooperative Soft Tissue Sarcoma Group and the Italian Cooperative Group, has improved treatment protocols, analyzed large-scale cohort outcomes, unified classifications and defined RMS risk factors (17).

However, despite such advances in H\&N RMS treatment, unfavorable prognostic factors such as parameningeal location or alveolar histopathological type reduce patient survival rates significantly (survival rates, 49 and $44 \%$, respectively) (5). Surgical procedures have had a notable influence on prognosis, with radical macro- and microscopic resection correlating strongly with higher survival rates; however, the treatment of patients with recurrent RMS remains challenging (4). Local control in the form of aggressive surgical resection and radiation therapy to sites not irradiated previously is generally recommended, particularly in patients with localized recurrence (4). Furthermore, there is no expert consensus on second- or third-line salvage chemotherapy to be used (4). The choice of chemotherapy is guided by the history of previous treatment received, and includes irinotecan with vincristine and temozolomide; topotecan with vincristine and doxorubicin; vinorelbine with oral cyclophosphamide; and gemcitabine with docetaxel $(4,18-21)$. For heavily pretreated patients, monotherapy of temsirolimus, bevacizumab, cediranib or cixutumumab has been proposed (4).

\section{Materials and methods}

Treatment setting. Retrospective analysis was performed on a cohort of 36 pediatric patients diagnosed with H\&N RMS who were treated at the Department of Otorhinolaryngology in the Faculty of Medicine and Dentistry of the Medical University of Warsaw (Warsaw, Poland) from January 2000 to December 2013. All patients underwent treatment with current therapy protocols for RMS, according to the guidelines of the Polish Pediatric Solid Tumors Group $(15,16,22)$. Therapy was conducted in collaboration with pediatric oncologists who supervised the treatment regime. Diagnostic biopsies and primary tumor resections were performed, as well as secondary resections after tumor relapse or after not achieving radical resection during previous surgery performed at other institutions. The primary surgical objective was tumor resection with negative margins, and obtaining acceptable cosmetic and functional outcomes. The histopathological specimens acquired during surgery were examined as indicated by the Polish Pediatric Solid Tumors Group (22). After surgery, patients returned to their referring oncology centers to continue therapy according to treatment protocol (chemo-/radiotherapy). Patients' treatment and outcomes were followed thereafter.

The patients' parameters included in the present analysis were age, gender, localization of the primary tumor, histopathological subtype, staging of the disease according to the tumor-node-metastasis (TNM) classification for RMS (23), treatment modalities and outcomes (Table I).

Ethics statement. All patients or their families provided written consent to the proposed treatment. The medical data used in the present study are anonymous and are presented in the form of cumulative statistical analysis, without photos or personal information that could allow the identification of each individual.

\section{Results}

Patient inclusion. From January 2000 to December 2013, 36 pediatric patients with H\&N RMS underwent surgical treatment in the Department of Otorhinolaryngology at the Medical University of Warsaw. The cohort consisted mostly of males (67\%) (Fig. 1). The mean age at the time of diagnosis was 7 years, and the patient age ranged from 21 months to 22 years (Fig. 2).

Cancer localization. A total of 24 patients (67\%) developed RMS in the parameningeal region. RMS primarily infiltrated the orbital region in 3 patients $(8 \%)$. In 9 other cases $(25 \%)$, 
Table I. Patients' characteristics and outcome.

\begin{tabular}{|c|c|c|c|c|c|c|}
\hline Pt no. & $\begin{array}{c}\text { Age at } \\
\text { diagnosis } \\
\text { (years) }\end{array}$ & $\begin{array}{l}\text { Pre-treatment } \\
\text { TNM staging }\end{array}$ & Localization & $\begin{array}{l}\text { Histological } \\
\text { subtype }\end{array}$ & Clinical course & Survival \\
\hline 1 & 10 & 3 & Parameningeal & ARMS & Local recurrence & Died 33 months post-ID \\
\hline 2 & 3 & 4 & Parameningeal & ERMS/ARMS & & Alive 11 years post-ID \\
\hline 3 & 4 & 3 & Other H\&N & ERMS & Local recurrence & Died 20 months post-ID \\
\hline 4 & 2 & 3 & Parameningeal & ERMS & & Alive 6 years post-ID \\
\hline 5 & 3 & 4 & Parameningeal & ERMS & No clinical remission & Died 24 months post-ID \\
\hline 6 & 4 & 3 & Parameningeal & ERMS & & Alive 8 years post-ID \\
\hline 7 & 8 & 3 & Parameningeal & ERMS/ARMS & & Alive 11 years post-ID \\
\hline 8 & 13 & 3 & Parameningeal & ERMS & Local recurrence & Alive 6 years post-ID \\
\hline 9 & 9 & 3 & Parameningeal & ERMS & & Alive 9 years post-ID \\
\hline 10 & 7 & 3 & Parameningeal & ERMS & Local recurrence & Died 33 months post-ID \\
\hline 11 & 6 & 3 & Parameningeal & ERMS & Local recurrence & Died 31 months post-ID \\
\hline 12 & 4 & 3 & Parameningeal & ERMS & & Alive 13 years post-ID \\
\hline 13 & 22 & 3 & Parameningeal & ERMS & Local recurrence & Died 31 months post-ID \\
\hline 14 & 1 & 3 & Parameningeal & ERMS & Local recurrence & Died 37 months post-ID \\
\hline 15 & 10 & 3 & Parameningeal & ERMS & & Alive 7 years post-ID \\
\hline 16 & 2 & 1 & Other H\&N & ARMS & Local recurrence & Alive 8 years post-ID \\
\hline 17 & 7 & 3 & Parameningeal & ERMS & & Alive 12 years post-ID \\
\hline 18 & 4 & 3 & Parameningeal & ERMS & Local recurrence & Died 27 months post-ID \\
\hline 19 & 5 & 4 & Parameningeal & ARMS & Local recurrence & Died 42 months post-ID \\
\hline 20 & 4 & 3 & Parameningeal & ERMS/ARMS & No clinical remission & Died 11 months post-ID \\
\hline
\end{tabular}

Pt no, patient number; TNM, tumor-node-metastasis; H\&N, head and neck; ARMS, alveolar rhabdomyosarcoma; ERMS, embryonal rhabdomyosarcoma; ID, initial diagnosis.

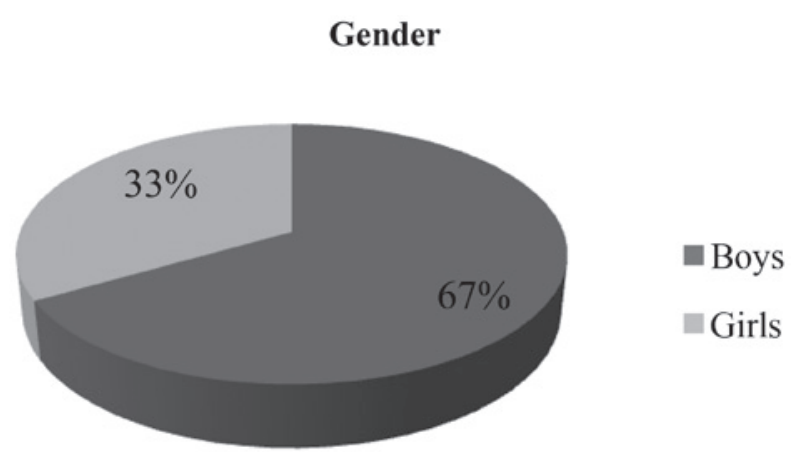

Figure 1. Distribution of patients according to gender.

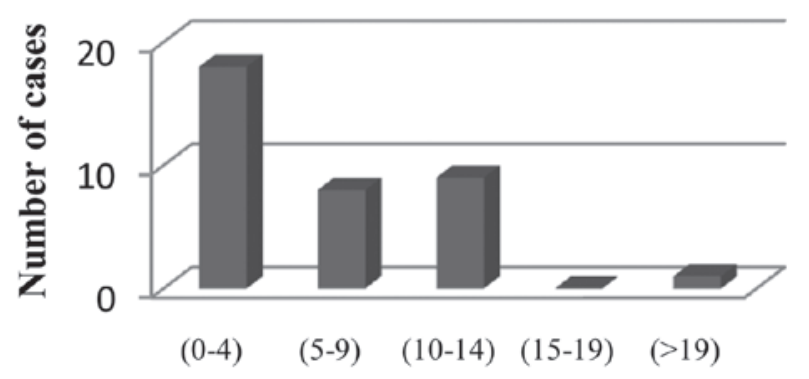

Age (years)

Figure 2. Distribution of patients according to age. the cancer was localized in different regions of the H\&N (Fig. 3).

Cancer histopathology. The most commonly observed RMS histopathology was embryonal subtype (28 cases, 78\%).

In 5 patients $(14 \%)$ alveolar subtype of RMS was diagnosed, and in other 3 patients $(8 \%)$, tumor tissue consisted of both alveolar and embryonal (mixed alveolar/embryonal RMS) (Fig. 4).

Cancer diagnosis. Only 2 patients had their primary diagnostic biopsies performed in the Czerniakowski Hospital (Warsaw,
Poland), while 16 patients were referred to the Department of Otorhinolaryngology at the Czerniakowski Hospital due to cancer relapse or previous non-radical surgical treatment. Of them, 75\% had previously undergone surgery for RMS. A total of 18 patients were referred to the Czerniakowski Hospital for primary surgical treatment following induction chemotherapy, with radiotherapy if required.

Cancer stage. In the analyzed cohort, the majority of patients presented with advanced disease. Among the patients referred to the Department of Otorhinolaryngology at the Czerniakowski Hospital for primary surgical treatment, 


\section{Localization}

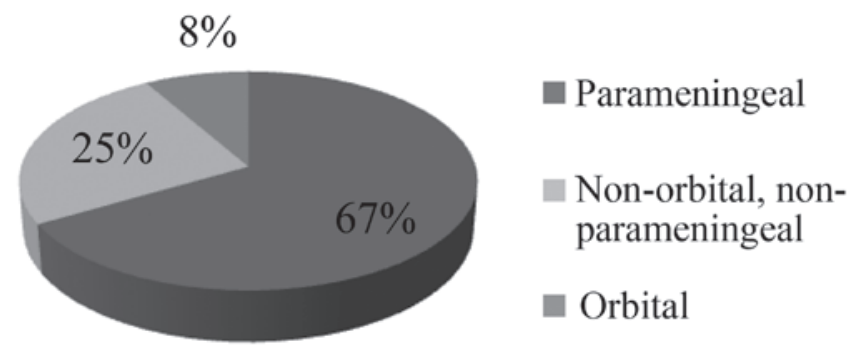

Figure 3. Distribution of patients according to the primary tumor site.

\section{Histology}

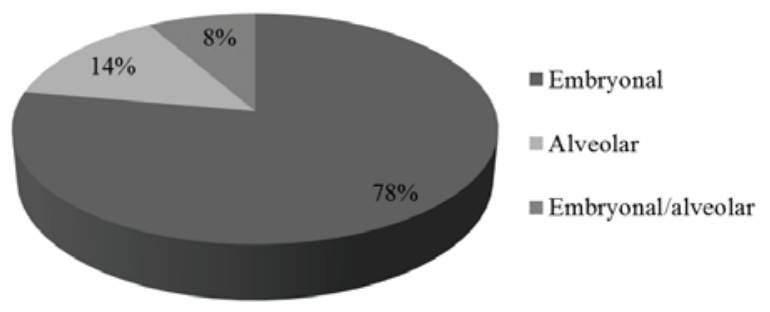

Figure 4. Distribution of patients according to histological subtype.

16 had stage III RMS disease according to TNM classification, 3 patients presented with lung metastasis, and 1 patient had bone marrow involvement.

Surgical treatment. Lateral, anterior, basal-through and combined surgical approaches were used depending on the localization and extent of disease progression. The most common localization was the skull base, and craniotomy was the most common surgical approach (20 cases). One patient had a concurrent nasal cavity surgical approach, while 2 others had a concurrent pharyngotomy. In 9 cases, access was achieved through rhinotomy with partial maxillectomy, if required; 2 cases involved a sublabial approach; 1 patient underwent petrosectomy; and 3 patients required lymphadenectomy due to disease stage. In other cases, different surgical procedures, including parotidectomy and resection of tumor of the parapharyngeal space or submandibular region, were performed if necessary.

Early complications manifested as 1 case of hematoma in the postoperative site, which required surgical intervention, and 1 case of cutaneous flap necrosis. Among the late complications, there were 3 cases of trismus after orbitozygomatic craniotomy; rehabilitation relieved symptoms in 2 of these patients, while 1 patient required surgical treatment. Hypernasal speech developed in 2 other cases, caused by losses of soft palate tissue. Salivary fistula ending in the external acoustic meatus formed in 1 patient. The majority of children suffered from various grade cosmetic defects, which depended on the extent of surgical resection. In total, 4 patients had to undergo orbital exenteration due to tumor infiltration of the orbit and eyeball. In 2 cases, the orbital content, including the bony structures, was removed, and microvascular flap reconstruction was performed to close the postoperative site. One patient underwent reconstructive surgery to enable the usage of oculi prosthesis. In 1 case, reconstructive surgery in the orbital region was postponed until achieving complete clinical remission.

Treatment outcomes. Out of 20 patients treated with primary surgical resection, 10 patients succumbed to disease, 8 of them due to a disease relapse, while 2 others did not achieve clinical remission. Of the remaining patients, 2 were lost on follow-up, and 7 patients are currently free of disease with relapse-free survival at 6-13 years post-diagnosis. At present, 1 patient is treated due to a disease relapse. The 5-year OS for patients treated with primary surgical resection was estimated to be $50 \%$ (Fig. 5). In the cohort of 16 patients referred to the Czerniakowski Hospital to treat RMS relapse, 12 patients succumbed to disease, 1 patient was lost during follow-up and 3 patients remain in remission 8 years and 3 months on average after the procedure. The 5-year OS for those patients was estimated to be $46.67 \%$ (Fig. 6).

\section{Discussion}

The treatment strategies for children with H\&N RMS have changed drastically over the last 30 years (11). Until the early 1960s, the gold standard of treatment was primary surgical resection with possible irradiation of the postoperative site in cases of non-radical procedure or infiltration of surgical margins (24). This approach resulted in disturbingly low survival rates $(5-9 \%)(25,26)$. The introduction of the first chemotherapeutics revolutionized soft tissue sarcoma treatment (25). Long-standing comparative analysis of numerous subsequent treatment protocols resulted in defining combination therapy (multidrug chemotherapy with radiotherapy and surgery) as the gold standard of treatment $(9,13)$. RMS requires multidisciplinary care, and all patients with RMS should be placed on protocol-driven therapy (9). The protocol must define the timing of chemotherapy, radiation therapy and surgery (9). Whether complete surgical excision is feasible, it may be performed prior to the initiation of chemotherapy or upon induction treatment (9). Surgery should be determined by an experienced pediatric H\&N surgeon (9). The necessity for radiotherapy is based on the site of the primary tumor and the completeness of surgical excision (9). At present, the surgeon is a vital member of a multidisciplinary team that plans individual treatment for every RMS patient (14). Surgical duties include assessing preoperative classification, performing proper biopsies, planning and conducting surgical resections with consideration of chemo- and radiotherapy, and evaluating postoperative classification (14). The essential aim of surgical treatment is the complete and radical resection of the primary RMS tumor with an acceptable functional and cosmetic result (27). Radical resection with histopathologically confirmed cancer-free surgical margins warrants a favorable prognosis $(16,28)$. Surgical procedures play a major role in controlling cancer locally, particularly in patients who, due to their age ( $<2$ years), are disqualified from irradiation $(11,27)$. Recommendations from different study groups regarding the range of resection and optimal resection 


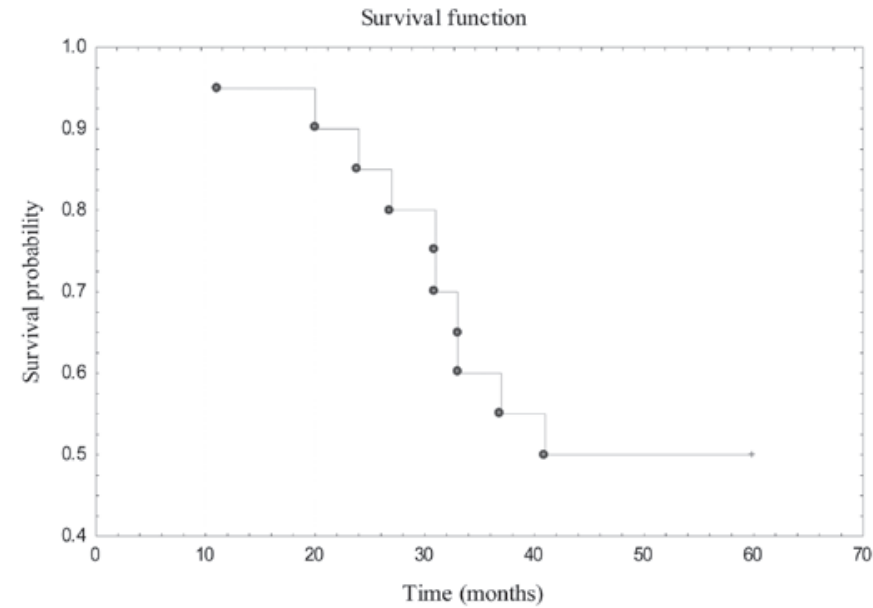

Figure 5. Kaplan-Meier survival curve showing the 5-year overall survival probability in the group of patients subjected to primary surgical treatment.

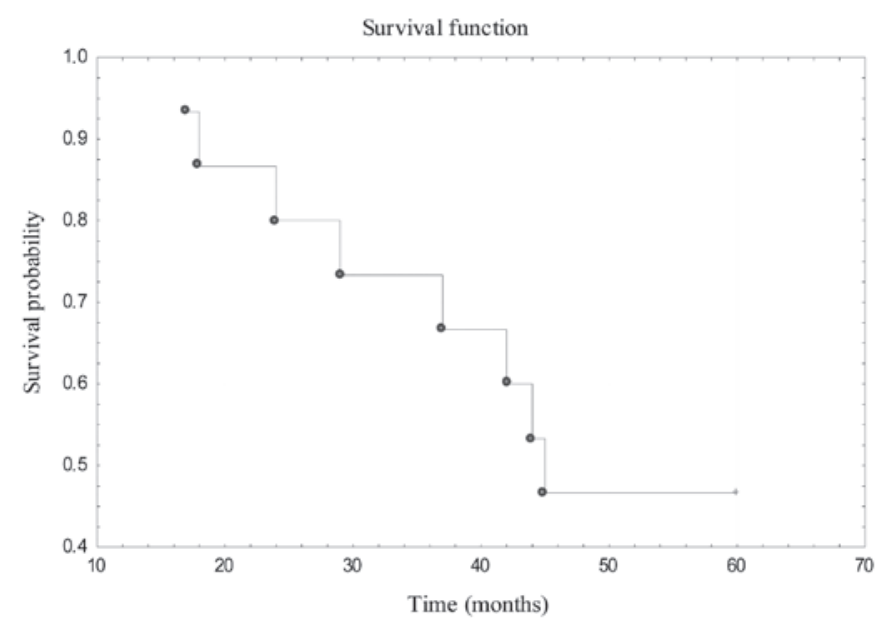

Figure 6. Kaplan-Meier survival curve showing the 5-year overall survival probability in the group of patients operated due to a disease relapse.

time varies substantially, and are under current analysis and discussion (27). It is also clear that when considering surgical treatment, the patient benefits from treatment in a large center where there is full access to a broad expertise, including a full preoperative assessment with diagnostic imaging to determine if there is bone erosion, intracranial spread or metastatic disease, which would be contraindications for surgery (29).

The signs and symptoms of pediatric H\&N RMS vary widely with these sites of involvement (8). Orbital and eyelid RMSs are readily identified, thereby facilitating early diagnosis and treatment (8). Children may demonstrate mild facial asymmetry, nasal congestion and serous otitis media. If RMS is localized in the paranasal sinuses, extensive growth prior to identification is possible, thus initiation of treatment may be delayed, and infiltration of adjacent vital structures may further complicate the management of these patients (8). Localization of H\&N RMS parameningeally or in the skull base also remains a challenge for surgeons (11). Sarcomas located parameningeally grow relatively large without any signs or symptoms, which delays treatment introduction $(8,10)$. Therefore, the primary tumor size often makes radical resec- tion impossible (8). Parameningeal RMS tends to infiltrate the skull base and expand intracranially, which worsens prognosis (1). Complicated skull base anatomy as well as plurality of important vital and functional anatomical structures limits surgical procedures, including impeded choice of optimal surgical approach, impossibility to resect the tumor en block, risk of losing important functional performance, inability to achieve acceptable cosmetic effect and difficulties at maintaining cancer-free surgical margins (30). All these properties may cause poor prognosis, and explain the necessity for more aggressive systemic treatment in patients with parameningeal RMS (1). Surgical treatment of malignant neoplasia originating in the skull base requires great experience and collaboration of different specialists, including H\&N surgeons, neurosurgeons, oral and maxillofacial surgeons, and plastic surgeons (14). Therefore, only selected medical centers offer such treatment. Primary RMS site in the orbital region and other more accessible regions of the $\mathrm{H} \& \mathrm{~N}$ provides favorable prognosis (with exclusion of parameningeal location, as aforementioned), since it involves earlier symptoms occurrence, facilitates decision-making regarding implementation of further diagnostic imaging [such as computed tomography (CT) and magnetic resonance imaging (MRI)] and decreases time to surgery (30).

Children with localized H\&N RMS are able to undergo complete surgical resection with low long-term surgical morbidity (31). It was reported that, by undergoing complete surgical resection, these patients avoid radiotherapy and its long-term complications, with no compromise in survival (31). Patients in whom radical resection with healthy tissues margin is impossible should receive neoadjuvant chemotherapy (27,32). A postponed procedure must be performed upon achieving satisfactory response to chemotherapy (32). Second-look surgery must be undertaken to resect viable tumors after the administration of definitive local therapy (1). In the IRS III, patients underwent induction chemotherapy and radiotherapy prior to second-look surgery $(33,34)$. Surgery was delayed as much as 20 weeks. In that trial, $52 \%$ of patients undergoing secondary operations, upon achieving only a minor response, were converted to a complete remission status by surgery $(33,34)$.

According to the IRS III, $>1 / 2$ of patients with localized disease had previously undergone only subtotal resection or biopsy as a surgical treatment (33). Patients classified after surgical intervention in this group were at higher risk of cancer relapse than patients with completely resected localized tumor or tumor grossly resected with the evidence of microscopic residual disease only (33). Since the majority of treatment failures apply to patients with relapse in the primary site, radiotherapy plays a substantial role in local RMS treatment. In addition, involvement of lymph nodes at primary diagnosis predicts a higher risk of local and distant treatment failure compared with children with negative lymph nodes (35). Smith et al (28) suggested the use of radiation therapy for every patient with confirmed microscopically cancer remnants, affection of regional lymph nodes and RMS histopathological subtype linked with unfavorable prognosis. Furthermore, according to a report by the Polish Pediatric Solid Tumors Group, radiotherapy should be used in every patient with parameningeal RMS (15).

Since survival rates in patients with RMS have improved with more effective treatment regimens, more long-term treatment-associated complications have been described (8). 
The most frequent difficulties are associated with radiation exposure, and occasional problems result from the cytotoxic effects of chemotherapy (8). The most frequently identified difficulties involve speech and memory skills (8). Other common complications include facial and statural growth retardation, neuroendocrine dysfunction, visual or orbital difficulties, hearing loss, intellectual and academic delays, and development of secondary malignancies (8). In RMS survivors, secondary leukemias were linked to etoposide therapy, and bone sarcomas in the sites of radiation treatment have been reported (35). In terms of otorhinolaryngology and $\mathrm{H} \& \mathrm{~N}$ surgery long-term care, H\&N RMS treatment may result in dentofacial abnormalities that affect the patient's quality of life (29). In patients with H\&N RMS who were followed at the Dental Service of the Memorial Sloan Kettering Cancer Center (New York City, NY, USA) and were alive and free of disease with $\geq 5$-year follow-up, multiple clinical and radiographic dentofacial abnormalities were observed, including facial asymmetry, enamel defects, bony hypoplasia, trismus, velopharyngeal insufficiency, tooth and root agenesis, malformed or missing teeth, microdontia, maxillary and mandibular hypoplasia, disturbance in root development, poor tooth development, root stunting and xerostomia $(8,29)$. The care of the long-term survivor requires a multidisciplinary approach, including early involvement of the dental specialists (29). Furthermore, behavioral problems, including hyperactivity, depression, immaturity and suicidal behaviors, have also been observed in certain RMS patients (8). The majority of RMS complications are encountered within the first 10 years after radiotherapy and chemotherapy (8).

A limited number of studies in the literature comment on the surgical treatment of pediatric H\&N RMS. The majority of publications concern population studies, epidemiological analyses or multinational collaborations summarizing the efficacy of combined therapy treatment according to differing treatment protocols (13).

Moretti et al (36), from the Otorhinolaryngological Unit at the Clinical Hospital of The University of São Paulo Medical School (São Paulo, Brazil), described a group of 24 patients suffering from RMS of the H\&N. Similar to the present study, the majority of cases were parameningeal RMS, but only 4 patients received surgical treatment as a part of combined therapy, and in 2 cases, resection was not radical. Fyrmpas et al (37) reported 14 patients with RMS of the nasal area and paranasal sinuses. Among them, 6 underwent primary surgical resections with subsequent chemotherapy and, if required, radiotherapy. Surgical approaches included combined approach (endoscopic and external) in 3 cases, sublabial approach (mid-facial degloving) in 2 cases and intranasal approach in 1 case. Resection was not radical in 2 cases. Only 1 patient suffered from progressive disease and consequently succumbed to disease (37).

In the current study, a heterogenous patient cohort that was treated at Department of Otorhinolaryngology at the Czerniakowski Hospital is presented. Among these patients, $\sim 1 / 2$ of them were referred due to previous treatment failure for salvage surgery. A significant number of tumors were parameningeal, which is an anatomical space difficult for surgical access and correlated with an unfavorable long-term prognosis. Out of 12 patients with a favorable prognosis due to tumor localization, 10 were operated in the Department of Otorhinolaryngology at the Czerniakowski Hospital due to cancer relapse, 9 of whom had previously undergone surgical treatment in different medical centers. The majority of patients (18 in total) had locally advanced cancer after induction chemotherapy and, possibly, radiotherapy. In the majority of cases, residual tumor was localized in the surrounding area of the infratemporal and pterygopalatine fossa; thus, the preferable surgical approach orbitozygomatic craniotomy. Skin incision was performed in line with the coronal suture, and subsequently lengthened longitudinally down along the anterior border of the auricle. This minimized postoperative scarring. Revelation of skin, fascial layer, temporal muscle and zygomatic arch excision provided excellent access to the infratemporal fossa, which allowed the identification and occasional preservation of the trigeminal nerve and maxillary artery. Furthermore, it eased complete resection of the pterygoid muscles with their bone insertion (pterygoid processes, temporomandibular joint and part of the mandibular ramus) in cases of muscle infiltration. The unilateral resection of the temporomandibular joint caused minor face asymmetry, and led to trismus in 3 cases, 1 of who required surgical correction while 2 others only underwent rehabilitation.

In 9 patients with RMS localized in the limits of the nasopharynx and/or maxillo-ethmoidal complex, the preferred surgical approach was lateral rhinotomy with partial maxillectomy. This approach allowed access into the nasal cavity structures, and following excision of the medial part of the maxilla with frontal process, it enabled the revision of the entire maxillary sinus. Furthermore, acceptable exposition of the ethmoid bone granted insight into possible infiltration towards the base of the anterior crania fossa.

Independently from the surgical approach, macroscopic assessment of the tumor infiltration margins was difficult, and intraoperative histopathological examination of the surgical margins previously altered by radiochemotherapy was not always evident. Due to those limitations, the scope of operation relied mainly on the result from imaging studies (CT and MRI). Adequate assessment of tissue material acquired during surgery required specific experience and often involved immunohistochemical staining; thus, the reference center evaluated and verified every histopathological result. After surgery was performed at the Department of Otorhinolaryngology at the Czerniakowski Hospital, patients continued therapy according to treatment protocols at their respective referring oncology centers. Patients were postoperatively monitored, due to collaboration between the Department of Otorhinolaryngology of the Faculty of Medicine and Dentistry of the Medical University of Warsaw and the aforementioned oncology centers providing subsequent therapy. However, not all patients reported for follow-up, mainly due to the distance from their place of residence and ongoing systemic therapy. The relatively low survival rate in the group of patients operated at the primary treatment stage may result from the advanced stage of the disease at the moment of diagnosis (95\% of patients exhibited stage $\geq 3$ ) and unfavorable tumor localization ( $86 \%$ of the group). In the group operated due to a disease relapse, the tumor was localized mainly in a favorable site, thus resulting in a relatively good survival rate. 
It should be emphasized that management of H\&N RMS must be interdisciplinary at the time of diagnosis. The surgical approach should be based on the individual characteristics of each patient. It is important to explain the possible complications of surgery, such as functional losses, cosmetic defects and permanent limitations, to parents and to the child, if necessary. In case of parameningeal RMS, obtaining negative surgical margins is often impossible; therefore, subsequent adjuvant therapy is indispensable. Experienced pathologists should examine both tumor en block and intraoperative tissue samples to assess the effectiveness of the surgical treatment. Surgeons should provide radiologists evaluating postsurgical CT or MRI scans with specific information regarding the surgical procedure and descriptions on how the operative site was reconstructed, as close collaboration in this field prevents misdiagnosis of disease relapse, which could lead to needless reoperations. ${ }^{18} \mathrm{~F}$-fluorodeoxyglucose positron emission tomography/CT should become a part of the standard diagnostic algorithm for children diagnosed with RMS, although dedicated bone imaging with ${ }^{99} \mathrm{Tc}$-methylene diphosphonate bone imaging may not be necessary in these patients. To reduce the radiation exposure of pediatric RMS patients to a minimum, as demanded by the As Low As Reasonably Achievable principle $(38,39)$, the number of imaging procedures should be as low as possible, since children are 10-15 times more sensitive to radiation than adults (40). Furthermore, children with RMS are often under surveillance for numerous years with serial CT imaging; thus, the development of novel imaging approaches to reduce radiation exposure is required (41). The role of second-look surgery after chemotherapy and radiation remains unknown, and trials must be designed in order to develop guidelines for such patients. It is likely that molecular biology research exploring the basic genetic mechanisms of RMS tumorigenesis and metastases development in the future will lead to the development of novel treatment strategies. Immunotherapy, targeted therapy and anti-angiogenic agents are potential new therapy modalities in pediatric H\&N RMS (35).

\section{Acknowledgements}

The present study was supported by the Medical University of Warsaw (Warsaw, Poland) statutory funding and the Military Institute of Medicine (Warsaw, Poland) statutory funding. Treatment costs were covered by the Polish National Health Fund (Warsaw, Poland). A.M.C. was supported by a SONATA grant from the National Science Centre (NCN; Warsaw, Poland; grant no. UMO-2012/05/D/NZ5/01844) and a WIM intramural grant from the Military Institute of Medicine [grant no. 1/8863 (355)]. A.M.C and C.S. were supported by an OPUS grant from the NCN (grant no. UMO-2011/01/B/NZ5/02822).

\section{References}

1. Dasgupta R and Rodeberg DA: Update on rhabdomyosarcoma. Semin Pediatr Surg 21: 68-78, 2012

2. Huh WW and Skapek SX: Childhood rhabdomyosarcoma: New insight on biology and treatment. Curr Oncol Rep 12: 402-410, 2010

3. Leaphart $C$ and Rodeberg D: Pediatric surgical oncology: Management of rhabdomyosarcoma. Surg Oncol 16: 173-185, 2007.
4. Ray A and Huh WW: Current state-of-the-art systemic therapy for pediatric soft tissue sarcomas. Curr Oncol Rep 14: 311-319, 2012.

5. Turner JH and Richmon JD: Head and neck rhabdomyosarcoma: A critical analysis of population-based incidence and survival data. Otolaryngol Head Neck Surg 145: 967-973, 2011.

6. Pappo AS, Shapiro DN, Crist WM and Maurer HM: Biology and therapy of pediatric rhabdomyosarcoma. J Clin Oncol 13: 2123-2139, 1995 .

7. Miller RW, Young JL Jr and Novakovic B: Childhood cancer. Cancer 75 (Suppl 1): S395-S405, 1995.

8. Holsinger FC, Weeks BH, Hicks MJ and Friedman EM: Contemporary concepts in the management of pediatric rhabdomyosarcoma. Curr Opin Otolaryngol Head Neck Surg 10: 91-96, 2002.

9. Hayes-Jordan A and Andrassy R: Rhabdomyosarcoma in children. Curr Opin Pediatr 21: 373-378, 2009.

10. Ahmed AA and Tsokos M: Sinonasal rhabdomyosarcoma in children and young adults. Int J Surg Pathol 15: 160-165, 2007.

11. Stevens MC: Treatment for childhood rhabdomyosarcoma: The cost of cure. Lancet Oncol 6: 77-84, 2005.

12. Wachtel M, Dettling M, Koscielniak E, Stegmaier S, Treuner J, Simon-Klingenstein K, Bühlmann P, Niggli FK and Schäfer BW: Gene expression signatures identify rhabdomyosarcoma subtypes and detect a novel $\mathrm{t}(2 ; 2)(\mathrm{q} 35 ; \mathrm{p} 23)$ translocation fusing PAX3 to NCOA1. Cancer Res 64: 5539-5545, 2004.

13. Gradoni P, Giordano D, Oretti G, Fantoni M and Ferri T: The role of surgery in children with head and neck rhabdomyosarcoma and Ewing's sarcoma. Surg Oncol 19: e103-109, 2010.

14. Rodeberg DA, Paidas CN, Lobe TL, Brown K, Andrassy RJ, Crist WM and Wiener ES: Surgical principles for children/adolescents with newly diagnosed rhabdomyosarcoma: A report from the Soft Tissue Sarcoma Committee of the Children's Oncology Group. Sarcoma 6: 111-122, 2002.

15. Zalewska-Szewczyk B, Kazanowska B, Mlynarski W, Dłużniewska A, Drożýnska E, Kurylak A, Nurzyńska-Flak J, Rybczyńska A, Pietniczka-Załęska M, Rychłowska-Pruszyńska M, et al: The analysis of clinical course and outcome of soft tissue sarcoma in parameningeal localization in children treated according to the CWS 96 protocol - a report of the Polish Paediatric Solid Tumours Group. Wspolczesna Onkol 12: 116-120, 2008.

16. Kazanowska B, Reich A, Reich M, Balcerska A, Balwierz W, Bodalski J, Dłuzniewska A, Drozyńska E, Katski K, Kijowski J, et al: Remaining problems and controversies in the management of childhood head and neck soft tissue sarcomas: Retrospective (national) Multicenter Study of the Polish Pediatric Solid Tumors Group. Pediatr Hematol Oncol 21: 349-362, 2004.

17. Radzikowska J, Kukwa W, Kukwa A, Czarnecka A and Krzeski A: Rhabdomyosarcoma of the head and neck in children. Contemp Oncol (Pozn) 19: 98-107, 2015.

18. McNall-Knapp RY, Williams CN, Reeves EN, Heideman RL and Meyer WH. Extended phase I evaluation of vincristine, irinotecan, temozolomide, and antibiotic in children with refractory solid tumors. Pediatr Blood Cancer 54: 909-915, 2010.

19. Wagner LM, Perentesis JP, Reid JM, Ames MM, Safgren SL, Nelson MD Jr, Ingle AM, Blaney SM and Adamson PC: Phase I trial of two schedules of vincristine, oral irinotecan, and temozolomide (VOIT) for children with relapsed or refractory solid tumors: A Children's Oncology Group phase I consortium study. Pediatr Blood Cancer 54: 538-545, 2010.

20. Casanova M, Ferrari A, Bisogno G, Merks JHM, De Salvo GL, Meazza C, Tettoni K, Provenzi M, Mazzarino I and Carli M: Vinorelbine and low-dose cyclophosphamide in the treatment of pediatric sarcomas: Pilot study for the upcoming European Rhabdomyosarcoma Protocol. Cancer 101: 1664-1671, 2004.

21. Rapkin L, Qayed M, Brill P, Martin M, Clark D, George BA, Olson TA, Wasilewski-Masker K, Alazraki A and Katzenstein HM: Gemcitabine and docetaxel (GEMDOX) for the treatment of relapsed and refractory pediatric sarcomas. Pediatr Blood Cancer 59: 854-858, 2012.

22. Kazanowska B and Chybicka A: Nowotwory tkanek miękkich. In: Zalecenia Postępowania Diagnostyczno - Terapeutycznego W Nowotworach Złośliwych. Krzakowski M (ed). 1st edition. Via Medica Gdansk, Gdańsk, pp868-893, 2012 (In Polish).

23. Lawrence W Jr, Anderson JR, Gehan EA and Maurer H; Children's Cancer Study Group: Pretreatment TNM staging of childhood rhabdomyosarcoma: A report of the Intergroup Rhabdomyosarcoma Study Group Pediatric Oncology Group. Cancer 80: 1165-1170, 1997. 
24. Dito WR and Batsakis JG: Rhabdomyosarcoma of the head and neck. An appraisal of the biologic behavior in 170 cases. Arch Surg 84: 582-588, 1962.

25. Healy GB, Upton J, Black PM and Ferraro N: The Role of surgery in rhabdomyosarcoma of the head and neck in children. Arch Otolaryngol Head Neck Surg 117: 1185-1188, 1991.

26. Masson JK and Soule EH: Embryonal rhabdomyosarcoma of head and neck. Report on eighty-eight cases. Am J Surg 110 585-591, 1965

27. Gosiengfiao Y, Reichek J and Walterhouse D: What is new in rhabdomyosarcoma management in children? Pediatr Drugs 14: 389-400, 2012.

28. Smith LM, Anderson JR, Qualman SJ, Crist WM, Paidas CN, Teot LA, Pappo AS, Link MP, Grier HE, Wiener ES, et al: Which patients with microscopic disease and rhabdomyosarcoma experience relapse after therapy? A report from the soft tissue sarcoma committee of the children's oncology group. J Clin Oncol 19: 4058-4064, 2001.

29. Estilo CL, Huryn JM,Kraus DH, Sklar CA, Wexler LH, Wolden SL and Zlotolow IM: Effects of therapy on dentofacial development in long-term survivors of head and neck rhabdomyosarcoma: The memorial sloan-kettering cancer center experience. J Pediatr Hematol Oncol 25: 215-222, 2003. Holsinger FC, Weeks BH, Hicks MJ and Friedman EM: Contemporary concepts in the management of pediatric rhabdomyosarcoma. Current Opinion in Otolaryngology \& Head and Neck Surgery 10: 91-96, 2002.

30. Dickson PV and Davidoff AM: Malignant neoplasms of the head and neck. Semin Pediatr Surg 15: 92-98, 2006.

31. Daya H, Chan HS, Sirkin W and Forte V: Pediatric rhabdomyosarcoma of the head and neck: Is there a place for surgical management? Arch Otolaryngol Head Neck Surg 126: 468-472, 2000.

32. Walterhouse D and Watson A: Optimal management strategies for rhabdomyosarcoma in children. Paediatr Drugs 9: 391-400, 2007.
33. Wharam MD, Meza J, Anderson J, Breneman JC, Donaldson SS, Fitzgerald TJ, Michalski J, Teot LA, Wiener ES and Meyer WH: Failure pattern and factors predictive of local failure in rhabdomyosarcoma: A report of group III patients on the third Intergroup Rhabdomyosarcoma Study. J Clin Oncol 22: 1902-1908, 2004.

34. Crist W, Gehan EA, Ragab AH, Dickman PS, Donaldson SS, Fryer C, Hammond D, Hays DM, Herrmann J, Heyn R, et al: The Third Intergroup Rhabdomyosarcoma Study. J Clin Oncol 13: 610-630, 1995.

35. Dagher R and Helman L: Rhabdomyosarcoma: An overview. Oncologist 4: 34-44, 1999.

36. Moretti G, Guimarães R, Oliveira KM, Sanjar F and Voegels RL Rhabdomyosarcoma of the head and neck: 24 cases and literature review. Braz J Otorhinolaryngol 76: 533-537, 2010 (In English, Portuguese).

37. Fyrmpas G, Wurm J, Athanassiadou F, Papageorgiou T, Beck JD, Iro $\mathrm{H}$ and Constantinidis J: Management of paediatric sinonasal rhabdomyosarcoma. J Laryngol Otol 123: 990-996, 2009.

38. McCarville MB: Imaging techniques used in the diagnosis of pediatric tumors. In: Pediatric Malignancies: Pathology and Imaging. Parham DM, Khoury JD and McCarville MB (eds). Springer Science and Business Media, New York, NY, pp7-18, 2015.

39. Brody AS, Frush DP, Huda W and Brent RL; American Academy of Pediatrics Section on Radiology. Radiation risk to children from computed tomography. Pediatrics 120: 677-682, 2007.

40. Brenner D, Elliston C, Hall E and Berdon W: Estimated risks of radiation-induced fatal cancer from pediatric CT. AJR Am J Roentgenol 176: 289-296, 2001.

41. Walter F, Czernin J, Hall T, Allen-Auerbach M, Walter MA, Dunkelmann S and Federman N: Is there a need for dedicated bone imaging in addition to $18 \mathrm{~F}-\mathrm{FDG} \mathrm{PET} / \mathrm{CT}$ imaging in pediatric sarcoma patients? J Pediatr Hematol Oncol 34: 131-136, 2012. 